\title{
Correlation transfer equation for multiply scattered light modulated by an ultrasonic pulse
}

\author{
Sava Sakadžić ${ }^{1,2}$ and Lihong V. Wang ${ }^{1,3, *}$ \\ ${ }^{1}$ Optical Imaging Laboratory, Department of Biomedical Engineering, Texas A\&M University, College Station, \\ Texas 77843-3120, USA \\ ${ }^{2}$ Present address, Massachusetts General Hospital, Athinoula A. Martinos Center for Biomedical Imaging, \\ Harvard Medical School, Charlestown, Massachusetts 02129, USA \\ ${ }^{3}$ Current address: Department of Biomedical Engineering, Washington University in St. Louis, Campus Box 1097, \\ One Brookings Drive, St. Louis, Missouri 63130-4899, USA \\ *Corresponding author: lhwang@biomed.wustl.edu
}

Received January 16, 2007; revised April 15, 2007; accepted April 16, 2007; posted April 17, 2007 (Doc. ID 79037); published August 8, 2007

\begin{abstract}
We develop a temporal correlation transfer equation (CTE) and a Monte Carlo algorithm (MC) for multiply scattered light modulated by an ultrasonic pulse propagating in an optically scattering medium, where the ultrasound field can be nonuniform and the medium can have spatially heterogeneous distribution of optical parameters. The CTE and MC can be used to obtain the time-varying specific intensity and the spatial distribution of the time-dependent power spectral density, respectively, of ultrasound-modulated light. We expect the CTE and MC to be applicable for estimation of contrast and resolution in a wide spectrum of conditions in ultrasound-modulated optical tomography of soft biological tissues. (C) 2007 Optical Society of America

OCIS codes: 030.1670, 030.5620, 170.7050, 170.6960, 290.4210, 290.7050.
\end{abstract}

\section{INTRODUCTION}

There is increasing interest in developing new imaging modalities based on the optical properties of soft biological tissues. At visible and near-infrared optical wavelengths the optical properties of biological tissues are related to the molecular structure. Radiation at these wavelengths is nonionizing with the potential for the detection of functions and abnormalities. However, it is difficult to obtain good spatial resolution at imaging depths greater than one optical transport mean free path owing to the strong diffusion of light at these wavelengths [1].

Ultrasound-modulated optical tomography (UOT) is a hybrid technique that combines the advantages of ultrasonic resolution and optical contrast [2,3]. In this technique, optical radiation of high temporal coherence and focused ultrasound are simultaneously applied to soft biological tissue. The resulting ultrasound-modulated light provides information about the optical properties of the tissue, spatially localized at the interaction region of the ultrasonic and the electromagnetic waves. UOT images that are based on the optical properties of a tissue sample are typically created by scanning either the ultrasound or the tissue sample $[4,5]$.

Owing to diffused light propagation and uncorrelated phases among optical speckles in UOT experiments, efficient detection of ultrasound-modulated light has proven to be challenging. At present, the development of effective detection techniques for UOT is the subject of intense research [3-17]. Simultaneously, a theoretical understanding of the ultrasound modulation of multiply scattered light is emerging. Present theoretical models include two mechanisms of modulation. The first mechanism accounts for dynamic scattering by optical scatterers oscillating in an ultrasound field, which causes optical frequency shifts $[6,18]$ as does dynamic light scattering by scatterers undergoing Brownian motion [19]. The second mechanism accounts for ultrasound-induced changes in the optical index of refraction [20,21]. The two mechanisms have been combined by Wang [21] in a model based on the diffusingwave spectroscopy (DWS) approach [19,22]. Subsequently, the equations are extended to account for anisotropic optical scattering [23] and Brownian motion [23,24]. In addition [25] accounts for pulsed ultrasound and strong correlations between ultrasound-induced optical phase increments, which exist when the ratio of the optical transport mean free path $l_{t r}$ to the ultrasound wavelength $\lambda_{a}$ is small. In the case of anisotropic optical scattering, equations derived for the isotropic case can be applied by substituting $l_{t r}$ for the optical mean free path $l_{s}[23,25]$.

The applications of theoretical models based on the DWS approach are limited to simple geometries where it is possible to approximate the ultrasound field within the sample with a plane ultrasound wave and where the probability density function of the optical path length between the source and detector is analytically known. In most experiments, however, the sample geometries are complex with heterogeneously distributed optical parameters, and a focused ultrasound beam is used.

Recently, a more general theoretical model based on the ladder diagram approximation of the Bethe-Salpeter equation [26-32] was developed [33,34]. A temporal correlation transfer equation (CTE) [35-38] was derived for multiply scattered light modulated by continuous wave (CW) ultrasound. This equation can be used to obtain the time-varying specific intensity and subsequently the power spectral density of light produced by a focused ul- 
trasound field in optically scattering media that have a heterogeneous distribution of optical parameters.

In addition, based on the theoretical models, Monte Carlo algorithms were developed and used for comparison with the theoretical predictions [23,25,33,34,39] as well as for modeling the scattering samples that have optically absorbing objects of cylindrical shapes [40].

In this paper, based on the ladder diagram approximation of the Bethe-Salpeter equation [26], we have derived a temporal correlation transfer equation (CTE) for multiply scattered light modulated by an ultrasonic pulse. The derivation is similar to the previous development of the CTE when CW ultrasound was used [34], and to the development of the CTE for scatterers moving with a given velocity distribution or undergoing Brownian motion [35-38]. In addition, it involves the link between the multiple scattering theory and the radiative transfer equation, which has been reviewed in numerous articles [27-32].

In Section 3, we develop a Monte Carlo simulation based on the CTE that can be used to calculate the timevarying power spectral density of light modulated by pulsed and focused ultrasound in optically turbid media with heterogeneous distributions of optical parameters. We further calculate the time-varying spatial distribution of the power spectral density of the ultrasound-modulated light produced by an ultrasound pulse with a center frequency of $1 \mathrm{MHz}$ that is focused in an optically scattering slab. The scattering slab has two embedded optically absorbing objects positioned within the ultrasonic focal zone with background optical parameters representative of those in soft biological tissues at visible and near-infrared wavelengths.

\section{DEVELOPMENT OF THE CTE}

The development of the CTE for multiply scattered light modulated by an ultrasound pulse is largely analogous to the derivation of the CTE for CW ultrasound [34]. The difference is that in the former case a more general representation of the spatiotemporal dependence of ultrasound is used. For the sake of clarity, we present the derivation steps with all details. We first develop an approximate expression for the Green's function of the electric field component in a medium free of optical scatterers and absorbers in the presence of an ultrasound field. For moderate ultrasound pressures, the optical index of refraction experiences a small perturbation and we locally approximate it with $n(\mathbf{r}, t)=n_{0}\left[1+\eta P(\mathbf{r}, t) /\left(\rho v_{a}^{2}\right)\right]$, where $n_{0}$ is the unperturbed optical index of refraction, $P(\mathbf{r}, t)$ is the ultrasound pressure, $\rho$ is the mass density of the medium, $v_{a}$ is the ultrasound speed, and $\eta$ is the elasto-optical coefficient (in water at standard conditions $v_{a} \approx 1480 \mathrm{~m} \mathrm{~s}^{-1}$, and $\eta \approx 0.32$ ).

We consider a source of monochromatic light having angular frequency $\omega_{0}$ and wave-vector magnitude $k_{0}=\omega_{0} / c_{0}$, where $c_{0}$ is the speed of light in vacuum. Time retardation and optical polarization effects are neglected for simplicity and we consider only one component of the electric field vector $\widetilde{E}(\mathbf{r}, t)$. Because of the large ratio of $\omega_{0}$ to the ultrasound angular frequency, we approximate the quasimonochromatic electric field in the medium as $\widetilde{E}(\mathbf{r}, t)$ $\approx E(\mathbf{r}, t) \exp \left(-i \omega_{0} t\right)$, where $E(\mathbf{r}, t)$ is a slowly changing function of time. For a point source of light positioned at $\mathbf{r}_{0}$, the slowly changing amplitude $E(\mathbf{r}, t)$ is given by the Green's function $G_{a}\left(\mathbf{r}, \mathbf{r}_{0}, t\right)$ that satisfies the equation

$$
\left[\nabla^{2}+k_{0}^{2} n_{0}^{2}\left(1+2 \frac{\eta P(\mathbf{r}, t)}{\rho v_{a}^{2}}\right)\right] G_{a}\left(\mathbf{r}, \mathbf{r}_{0}, t\right)=\delta\left(\mathbf{r}-\mathbf{r}_{0}\right),
$$

where $2 \eta|P(\mathbf{r}, t)| /\left(\rho v_{a}^{2}\right) \ll 1$, and $\delta()$ is the Dirac delta function.

We present $G_{a}\left(\mathbf{r}, \mathbf{r}_{0}, t\right)$ as

$$
G_{a}\left(\mathbf{r}, \mathbf{r}_{0}, t\right)=\frac{\exp \left(i k_{0} n_{0}\left|\mathbf{r}-\mathbf{r}_{0}\right|\left[1+\xi\left(\mathbf{r}, \mathbf{r}_{0}, t\right)\right]\right)}{-4 \pi\left|\mathbf{r}-\mathbf{r}_{0}\right|},
$$

where $\xi\left(\mathbf{r}, \mathbf{r}_{0}, t\right)$ is the small fractional phase perturbation that slowly varies in time and depends on $P(\mathbf{r}, t)$.

We further approximate $\xi\left(\mathbf{r}, \mathbf{r}_{0}, t\right)$ as

$$
\xi\left(\mathbf{r}, \mathbf{r}_{0}, t\right)=\frac{\eta}{\rho v_{a}^{2}\left|\mathbf{r}-\mathbf{r}_{0}\right|} \int_{\mathbf{r}_{0}}^{\mathbf{r}} P\left(\mathbf{r}^{\prime}, t\right) \mathrm{d} r^{\prime}
$$

where we assume moderate ultrasound pressures, distances $\mathbf{r}$ not far from the source position $\mathbf{r}_{0}$ such that $k_{0} n_{0}\left|\mathbf{r}-\mathbf{r}_{0}\right| \xi\left(\mathbf{r}, \mathbf{r}_{0}, t\right) \ll 1$, and that the ultrasound-induced refraction of the optical waves is negligible for the interaction length $\left|\mathbf{r}-\mathbf{r}_{0}\right|$.

Next, we consider independent optical scattering in an optically scattering medium representative of soft biological tissue having discrete and uncorrelated optical scatterers. We assume the weak scattering approximation that the optical mean free path $l_{s}$ is much greater than the optical wavelength $\lambda_{0}$. We also assume that the ultrasound field in volumes $\sim l_{t r}^{3}$ can be locally approximated as a plane wave $P(\mathbf{r}, t)=P_{0} h\left(t-v_{a} \hat{\mathbf{\Omega}}_{a} \cdot \mathbf{r}+\phi\right)$, where $h(x)$ is the propagation function of the ultrasound pulse normalized to unity, and $P_{0}, \phi$, and $\hat{\mathbf{\Omega}}_{a}$ are the local pressure amplitude, local initial phase, and local propagation direction unit vector of the ultrasound, respectively $\left(\left|\hat{\mathbf{\Omega}}_{a}\right|=1\right)$. Now, Eq. (3) could be expressed as

$$
\xi\left(\mathbf{r}, \mathbf{r}_{0}, t\right)=\frac{1}{2} M \int_{\mathbf{r}_{0}}^{\mathbf{r}} h\left(t-v_{a} \hat{\mathbf{\Omega}}_{a} \cdot \mathbf{r}^{\prime}+\phi\right) \mathrm{d} r^{\prime},
$$

where $M=2 \eta P_{0} /\left(\rho v_{a}^{2}\left|\mathbf{r}-\mathbf{r}_{0}\right|\right)$.

In further derivations, the expressions for $\xi\left(\mathbf{r}, \mathbf{r}_{0}, t\right)$ [Eq. (4)] and for $G_{a}\left(\mathbf{r}, \mathbf{r}_{0}, t\right)$ [Eq. (2)] are required to be approximately valid for $\left|\mathbf{r}-\mathbf{r}_{0}\right|$ on the order of a few $l_{t r}$, which is satisfied in soft biological tissues at visible and near-infrared optical wavelengths $\left(l_{t r} \approx 1 \mathrm{~mm}\right)$, for moderate ultrasound pressures $\left(P_{0} \leqslant 10^{5} \mathrm{~Pa}\right)$, and in the medical ultrasound frequency range [25].

The optical extinction, scattering, and absorption coefficients are defined as $\mu_{t}=\mu_{s}+\mu_{a}, \mu_{s}=\sigma_{s} \rho_{s}$, and $\mu_{a}=\sigma_{a} \rho_{s}$, respectively, where $\rho_{s}$ is the density of optical scatterers, $\sigma_{s}$ is the optical scattering cross section, and $\sigma_{a}$ is the optical absorption cross section. We assume that the optical scattering amplitude $f\left(\hat{\mathbf{\Omega}}_{\mathrm{sc}}, \hat{\mathbf{\Omega}}_{\text {inc }}\right)$ is a function of $\hat{\mathbf{\Omega}}_{\mathrm{sc}} \cdot \hat{\boldsymbol{\Omega}}_{\text {inc }}$ only, where $\hat{\boldsymbol{\Omega}}_{\text {inc }}$ and $\hat{\boldsymbol{\Omega}}_{\mathrm{sc}}$ are the directions of the incident and scattered waves, respectively. The scattering phase function $p\left(\hat{\boldsymbol{\Omega}}_{\mathrm{sc}}, \hat{\boldsymbol{\Omega}}_{\text {inc }}\right)$ is defined as $p\left(\hat{\boldsymbol{\Omega}}_{\mathrm{sc}}, \hat{\boldsymbol{\Omega}}_{\text {inc }}\right)$ 
$=\sigma_{s}^{-1}\left|f\left(\hat{\boldsymbol{\Omega}}_{\mathrm{sc}}, \hat{\boldsymbol{\Omega}}_{\mathrm{inc}}\right)\right|^{2}$, and it satisfies $\int_{4 \pi} p\left(\hat{\mathbf{\Omega}}_{\mathrm{sc}}, \hat{\boldsymbol{\Omega}}_{\mathrm{inc}}\right) \mathrm{d} \boldsymbol{\Omega}_{\mathrm{sc}}=1$. From the optical theorem we also have $\sigma_{s}+\sigma_{a}$ $=4 \pi \operatorname{Im}\left[f\left(\hat{\mathbf{\Omega}}_{\text {inc }}, \hat{\mathbf{\Omega}}_{\text {inc }}\right)\right] /\left(k_{0} n_{0}\right)$, where $\operatorname{Im}[]$ takes on the imaginary part.

The ensemble averaged value of the electric field at $\mathbf{r}_{b}$ emitted from a point source at $\mathbf{r}_{a}$ is referred to also as a mean or coherent field and it is provided by a mean Green's function $G_{s}\left(\mathbf{r}_{b}, \mathbf{r}_{a}, t\right)$. We assume sufficiently small optical scatterers and consider only the far-field approximations of the scattered fields. $G_{s}\left(\mathbf{r}_{b}, \mathbf{r}_{a}, t\right)$ is obtained by solving the Dyson equation [26,35,36], whose far-field expression in the Bourret approximation is given by

$$
\begin{aligned}
G_{s}\left(\mathbf{r}_{b}, \mathbf{r}_{a}, t\right)= & G_{a}\left(\mathbf{r}_{b}, \mathbf{r}_{a}, t\right)-4 \pi \rho_{s} \int G_{a}\left(\mathbf{r}_{b}, \mathbf{r}_{s}, t\right) f\left(\hat{\mathbf{\Omega}}_{s b}, \hat{\mathbf{\Omega}}_{a s}\right) \\
& \times \exp \left[i k_{0} n_{0} \mathbf{e}_{s}(t) \cdot\left(\hat{\boldsymbol{\Omega}}_{a s}-\hat{\mathbf{\Omega}}_{s b}\right)\right] G_{s}\left(\mathbf{r}_{s}, \mathbf{r}_{a}, t\right) \mathrm{d} \mathbf{r}_{s}
\end{aligned}
$$

The exponential factor on the right hand side of Eq. (5) accounts for the Doppler shift caused by the ultrasoundinduced movement of the scatterer, and the position of the scatterer at time $t$ is $\mathbf{r}_{s}+\mathbf{e}_{s}(t)$, where $\mathbf{r}_{s}$ is the resting position and $\mathbf{e}_{s}(t)$ is the small ultrasound-induced displacement. The refraction of the mean optical field that is due to the ultrasound is neglected and $\hat{\mathbf{\Omega}}_{a s}$ and $\hat{\mathbf{\Omega}}_{s b}$ are unity vectors in directions $\mathbf{r}_{s}-\mathbf{r}_{a}$ and $\mathbf{r}_{b}-\mathbf{r}_{s}$, respectively.

Equation (5) can be solved by applying the method of stationary phase in a way similar to the derivation presented in the Appendix of [34]. The mean Green's function is expressed as

$$
G_{s}\left(\mathbf{r}_{b}, \mathbf{r}_{a}, t\right)=\frac{\exp \left[i K\left(\mathbf{r}_{b}, \mathbf{r}_{a}, t\right)\left|\mathbf{r}_{b}-\mathbf{r}_{a}\right|\right]}{-4 \pi\left|\mathbf{r}_{b}-\mathbf{r}_{a}\right|},
$$

where

$$
K\left(\mathbf{r}_{b}, \mathbf{r}_{a}, t\right)=k_{0} n_{0}\left[1+\xi\left(\mathbf{r}_{b}, \mathbf{r}_{a}, t\right)\right]+2 \pi \rho_{s} f(\hat{\mathbf{\Omega}}, \hat{\mathbf{\Omega}}) /\left(k_{0} n_{0}\right) .
$$

$G_{s}\left(\mathbf{r}_{b}, \mathbf{r}_{a}, t\right)$ is expressed in Eq. (6) similarly as in [34] where CW ultrasound is considered. However, the expression for $\xi\left(\mathbf{r}_{b}, \mathbf{r}_{a}, t\right)$ in [34] is just a special case of the more general expression in Eq. (4). The term $\xi\left(\mathbf{r}_{b}, \mathbf{r}_{a}, t\right)$ in propagation constant $K\left(\mathbf{r}_{b}, \mathbf{r}_{a}, t\right)$ is related to the accumulated optical phase from $\mathbf{r}_{a}$ to $\mathbf{r}_{b}$ due to ultrasoundinduced changes in the optical index of refraction, and the term $2 \pi \rho_{s} f(\hat{\mathbf{\Omega}}, \hat{\mathbf{\Omega}}) /\left(k_{0} n_{0}\right)$ accounts for the multiple wave scattering from $\mathbf{r}_{a}$ to $\mathbf{r}_{b}$. In the absence of optical scatterers $\left(\rho_{s}=0\right), G_{s}\left(\mathbf{r}_{b}, \mathbf{r}_{a}, t\right)$ reduces to $G_{a}\left(\mathbf{r}_{b}, \mathbf{r}_{a}, t\right)$.

To obtain the time-varying power spectral density of multiply scattered light at time $t$, we present the mutual coherence function of the electric field component as

$$
\Gamma\left(\mathbf{r}_{a}, \mathbf{r}_{b}, t, \tau\right)=\left\langle E\left(\mathbf{r}_{a}, t-\tau / 2\right) E^{*}\left(\mathbf{r}_{b}, t+\tau / 2\right)\right\rangle,
$$

where $\mathbf{r}_{a}$ and $\mathbf{r}_{b}$ are two closely spaced points relative to the optical mean free path $l_{t}$, and \langle\rangle represents ensemble averaging. We further assume that $\Gamma\left(\mathbf{r}_{a}, \mathbf{r}_{b}, t, \tau\right)$ is a quasi-uniform function that, under the weak-scattering approximation, satisfies the ladder approximation of the Bethe-Salpeter equation [26,35,36,38] for moving scatterers,
$\Gamma\left(\mathbf{r}_{a}, \mathbf{r}_{b}, t, \tau\right)=\Gamma_{0}\left(\mathbf{r}_{a}, \mathbf{r}_{b}, t, \tau\right)$

$$
\begin{aligned}
& +\iint v_{s^{\prime}}^{a}(t-\tau / 2) v_{s^{\prime \prime}}^{b^{*}}(t+\tau / 2) \Gamma\left(\mathbf{r}_{s^{\prime}}, \mathbf{r}_{s^{\prime \prime}}, t, \tau\right) \\
& \times \rho\left(\mathbf{r}_{s^{\prime}}, t-\tau / 2 ; \mathbf{r}_{s^{\prime \prime}}, t+\tau / 2\right) \mathrm{d} \mathbf{r}_{s^{\prime}} \mathrm{d} \mathbf{r}_{s^{\prime \prime}} .
\end{aligned}
$$

In Eq. (7), $\mathbf{r}_{s^{\prime}}$ and $\mathbf{r}_{s^{\prime \prime}}$ are the positions of the same scatterer at times $t-\tau / 2$ and $t+\tau / 2$, respectively, and $\rho\left(\mathbf{r}_{s^{\prime}}, t\right.$ $\left.-\tau / 2 ; \mathbf{r}_{s^{\prime \prime}}, t+\tau / 2\right)$ is the probability density of finding the same scatterer $s$ at position $\mathbf{r}_{s^{\prime}}$ and time $t-\tau / 2$, and at position $\mathbf{r}_{s^{\prime \prime}}$ and time $t+\tau / 2$. The term $\Gamma_{0}\left(\mathbf{r}_{a}, \mathbf{r}_{b}, t, \tau\right)$ $=\left\langle E\left(\mathbf{r}_{a}, t-\tau / 2\right)\right\rangle\left\langle E^{*}\left(\mathbf{r}_{b}, t+\tau / 2\right)\right\rangle$ is the mutual coherence function of the coherent (unscattered) field.

We first define the spectral density $\widetilde{\Gamma}\left(\mathbf{r}_{c s}, \mathbf{q}^{\prime}, t, \tau\right)$ of $\Gamma\left(\mathbf{r}_{s^{\prime}}, \mathbf{r}_{s^{\prime \prime}}, t, \tau\right)$ as

$$
\widetilde{\Gamma}\left(\mathbf{r}_{c s}, \mathbf{q}^{\prime}, t, \tau\right)=(2 \pi)^{-3} \int \Gamma\left(\mathbf{r}_{c s}, \mathbf{r}_{d s}, t, \tau\right) \exp \left(-i \mathbf{q}^{\prime} \cdot \mathbf{r}_{d s}\right) \mathrm{d} \mathbf{r}_{d s},
$$

where $\mathbf{r}_{d s}=\mathbf{r}_{s^{\prime}}-\mathbf{r}_{s^{\prime \prime}}$ and $\mathbf{r}_{c s}=\left(\mathbf{r}_{s^{\prime}}+\mathbf{r}_{s^{\prime \prime}}\right) / 2$. By assuming the far-field approximation of the operators $v_{s^{\prime}}^{a}(t-\tau / 2)$ and $v_{s^{\prime \prime}}^{b *}(t+\tau / 2)$, the term $v_{s^{\prime}}^{a}(t-\tau / 2) v_{s^{\prime \prime}}^{b *}(t+\tau / 2) \Gamma\left(\mathbf{r}_{s^{\prime}}, \mathbf{r}_{s^{\prime \prime}}, t, \tau\right)$ in Eq. (7) can be written as an integral over all the spectral components of $\Gamma\left(\mathbf{r}_{s^{\prime}}, \mathbf{r}_{s^{\prime \prime}}, t, \tau\right)$ as [34]

$$
\begin{aligned}
v_{s^{\prime}}^{a}(t & -\tau / 2) v_{s^{\prime \prime}}^{b^{*}}(t+\tau / 2) \Gamma\left(\mathbf{r}_{s^{\prime}}, \mathbf{r}_{s^{\prime \prime}}, t, \tau\right) \\
\equiv & \int \frac{f\left(\hat{\mathbf{\Omega}}_{s^{\prime} a}, \hat{\boldsymbol{\Omega}}^{\prime}\right) f^{*}\left(\hat{\boldsymbol{\Omega}}_{s^{\prime \prime} b}, \hat{\mathbf{\Omega}}^{\prime}\right)}{\left|\mathbf{r}_{a}-\mathbf{r}_{s^{\prime}}\right|\left|\mathbf{r}_{b}-\mathbf{r}_{s^{\prime \prime}}\right|} \widetilde{\Gamma}\left(\mathbf{r}_{c s}, \mathbf{q}^{\prime}, t, \tau\right) \\
& \times \exp \left[i K\left(\mathbf{r}_{a}, \mathbf{r}_{s^{\prime}}, t-\tau / 2\right)\left|\mathbf{r}_{a}-\mathbf{r}_{s^{\prime}}\right|\right] \\
& \times \exp \left[-i K^{*}\left(\mathbf{r}_{b}, \mathbf{r}_{s^{\prime \prime}}, t+\tau / 2\right)\left|\mathbf{r}_{b}-\mathbf{r}_{s^{\prime \prime}}\right|\right] \exp \left(i \mathbf{q}^{\prime} \cdot \mathbf{r}_{d s}\right) \mathrm{d} \mathbf{q}^{\prime},
\end{aligned}
$$

where $\hat{\mathbf{\Omega}}^{\prime}=\mathbf{q}^{\prime} /\left|\mathbf{q}^{\prime}\right|$.

Next, we define vectors $\mathbf{r}_{c}=\left(\mathbf{r}_{a}+\mathbf{r}_{b}\right) / 2, \mathbf{r}_{d}=\mathbf{r}_{a}-\mathbf{r}_{b}$, and $\hat{\mathbf{\Omega}}=\left(\mathbf{r}_{c}-\mathbf{r}_{c s}\right) /\left|\mathbf{r}_{c}-\mathbf{r}_{c s}\right|$ in the center-of-gravity coordinate system. Since $\left|\mathbf{r}_{d}\right| \ll\left|\mathbf{r}_{c}-\mathbf{r}_{c s}\right|$ and $\left|\mathbf{r}_{d s}\right| \ll\left|\mathbf{r}_{c}-\mathbf{r}_{c s}\right|$, it is safe to assume that $f\left(\hat{\mathbf{\Omega}}_{s^{\prime} a}, \hat{\mathbf{\Omega}}^{\prime}\right) \approx f\left(\hat{\mathbf{\Omega}}, \hat{\mathbf{\Omega}}^{\prime}\right), f\left(\hat{\boldsymbol{\Omega}}_{s^{\prime \prime} b}, \hat{\mathbf{\Omega}}^{\prime}\right) \approx f\left(\hat{\mathbf{\Omega}}, \hat{\mathbf{\Omega}}^{\prime}\right)$, and

$$
\begin{gathered}
\left|\mathbf{r}_{a}-\mathbf{r}_{s^{\prime}}\right| \approx\left|\mathbf{r}_{c}-\mathbf{r}_{c s}\right|+\left(\mathbf{r}_{d}-\mathbf{r}_{d s}\right) \cdot \hat{\mathbf{\Omega}} / 2, \\
\left|\mathbf{r}_{b}-\mathbf{r}_{s^{\prime \prime}}\right| \approx\left|\mathbf{r}_{c}-\mathbf{r}_{c s}\right|-\left(\mathbf{r}_{d}-\mathbf{r}_{d s}\right) \cdot \hat{\mathbf{\Omega}} / 2 \\
\quad\left(\left|\mathbf{r}_{a}-\mathbf{r}_{s^{\prime}}\right|\left|\mathbf{r}_{b}-\mathbf{r}_{s^{\prime \prime}}\right|\right)^{-1} \approx\left|\mathbf{r}_{c}-\mathbf{r}_{c s}\right|^{-2},
\end{gathered}
$$

and part of the expression on the right hand side of Eq. (9) can be presented as

$$
\begin{aligned}
& \frac{f\left(\hat{\boldsymbol{\Omega}}_{s^{\prime} a}, \hat{\boldsymbol{\Omega}}^{\prime}\right) f^{*}\left(\hat{\boldsymbol{\Omega}}_{s^{\prime \prime} b}, \hat{\boldsymbol{\Omega}}^{\prime}\right)}{\left|\mathbf{r}_{a}-\mathbf{r}_{s^{\prime}}\right|\left|\mathbf{r}_{b}-\mathbf{r}_{s^{\prime \prime}}\right|} \exp \left[i K\left(\mathbf{r}_{a}, \mathbf{r}_{s^{\prime}}, t-\tau / 2\right)\left|\mathbf{r}_{a}-\mathbf{r}_{s^{\prime}}\right|\right. \\
& \left.\quad-i K^{*}\left(\mathbf{r}_{b}, \mathbf{r}_{s^{\prime \prime}}, t+\tau / 2\right)\left|\mathbf{r}_{b}-\mathbf{r}_{s^{\prime \prime}}\right|\right] \\
& =\sigma_{s} p\left(\hat{\mathbf{\Omega}}, \hat{\boldsymbol{\Omega}}^{\prime}\right)\left|\mathbf{r}_{c}-\mathbf{r}_{c s}\right|^{-2} \exp \left[i K_{r}\left(\mathbf{r}_{d}-\mathbf{r}_{d s}\right) \cdot \hat{\boldsymbol{\Omega}}-\mu_{t}\left|\mathbf{r}_{c}-\mathbf{r}_{c s}\right|\right] \\
& \quad \times \exp \left[i \Psi_{n}\left(\mathbf{r}_{a}, \mathbf{r}_{b}, \mathbf{r}_{s^{\prime}}, \mathbf{r}_{s^{\prime \prime}}, t, \tau\right)\right],
\end{aligned}
$$

where $K_{r}=n_{0} k_{0}+4 \pi \operatorname{Re}[f(\hat{\mathbf{\Omega}}, \hat{\mathbf{\Omega}})] \rho_{s} /\left(2 k_{0} n_{0}\right)$, and $\operatorname{Re}[]$ 
takes on the real part. The term $\Psi_{n}\left(\mathbf{r}_{a}, \mathbf{r}_{b}, \mathbf{r}_{s^{\prime}}, \mathbf{r}_{s^{\prime \prime}}, t, \tau\right)$ is the difference between the ultrasound-induced phase increments given by

$$
\begin{aligned}
\Psi_{n}\left(\mathbf{r}_{a}, \mathbf{r}_{b}, \mathbf{r}_{s^{\prime}}, \mathbf{r}_{s^{\prime \prime}}, t, \tau\right)= & k_{0} n_{0}\left|\mathbf{r}_{a}-\mathbf{r}_{s^{\prime}}\right| \xi\left(\mathbf{r}_{a}, \mathbf{r}_{s^{\prime}}, t-\tau / 2\right) \\
& -k_{0} n_{0}\left|\mathbf{r}_{b}-\mathbf{r}_{s^{\prime \prime}}\right| \xi\left(\mathbf{r}_{b}, \mathbf{r}_{s^{\prime \prime}}, t+\tau / 2\right),
\end{aligned}
$$

and it could be approximated by using the relations in Eq. (4) and Eq. (10) with $\Psi_{n}\left(\mathbf{r}_{c}, \mathbf{r}_{c s}, t, \tau\right)$, where

$$
\begin{aligned}
\Psi_{n}\left(\mathbf{r}_{c}, \mathbf{r}_{c s}, t, \tau\right)= & \frac{1}{2} k_{0} n_{0} M \int_{\mathbf{r}_{c s}}^{\mathbf{r}_{c}}\left[h\left(t-\tau / 2-v_{a} \hat{\mathbf{\Omega}}_{a} \cdot \mathbf{r}^{\prime}+\phi\right)\right. \\
& \left.-h\left(t+\tau / 2-v_{a} \hat{\mathbf{\Omega}}_{a} \cdot \mathbf{r}^{\prime}+\phi\right)\right] \mathrm{d} r^{\prime} .
\end{aligned}
$$

The probability density function $\rho\left(\mathbf{r}_{s^{\prime}}, t-\tau / 2 ; \mathbf{r}_{s^{\prime \prime}}, t\right.$ $+\tau / 2)$ in Eq. (7) can be expressed as $\rho_{s} \delta\left[\mathbf{r}_{d s}-\Delta \mathbf{e}\left(\mathbf{r}_{s}, t, \tau\right)\right]$, where $\Delta \mathbf{e}\left(\mathbf{r}_{s}, t, \tau\right)=\mathbf{e}_{s}(t-\tau / 2)-\mathbf{e}_{s}(t+\tau / 2)$, and the positions $\mathbf{r}_{s^{\prime}}$ and $\mathbf{r}_{s^{\prime \prime}}$ of the scatterer at times $t-\tau / 2$ and $t+\tau / 2$ are given by $\mathbf{r}_{s^{\prime}}=\mathbf{r}_{s}+\mathbf{e}_{s}(t-\tau / 2)$ and $\mathbf{r}_{s^{\prime \prime}}=\mathbf{r}_{s}+\mathbf{e}_{s}(t+\tau / 2)$, respectively.

Then we replace the integral in Eq. (7) over positions $\mathbf{r}_{s^{\prime}}$ and $\mathbf{r}_{s^{\prime \prime}}$ with an integral over $\mathbf{r}_{d s}$ and $\mathbf{r}_{c s}$. By using $\mathrm{d} \mathbf{r}_{c s}=\left|\mathbf{r}_{c}-\mathbf{r}_{c s}\right|^{2} \mathrm{~d}\left|\mathbf{r}_{c}-\mathbf{r}_{c s}\right| \mathrm{d} \Omega$ and by performing an additional integration over $\mathbf{r}_{d s}$, we have

$$
\begin{aligned}
\Gamma\left(\mathbf{r}_{c}, \mathbf{r}_{d}, t, \tau\right)= & \Gamma_{0}\left(\mathbf{r}_{c}, \mathbf{r}_{d}, t, \tau\right)+\int \mu_{s} p\left(\hat{\mathbf{\Omega}}, \hat{\mathbf{\Omega}}^{\prime}\right) \\
& \times \exp \left(i K_{r} \mathbf{r}_{d} \cdot \hat{\mathbf{\Omega}}\right) \exp \left(-\mu_{t}\left|\mathbf{r}_{c}-\mathbf{r}_{c s}\right|\right) \\
& \times \exp \left[i\left(\mathbf{q}^{\prime}-K_{r} \hat{\mathbf{\Omega}}\right) \cdot \Delta \mathbf{e}\left(\mathbf{r}_{s}, t, \tau\right)\right] \\
& \times \exp \left[i \Psi_{n}\left(\mathbf{r}_{c}, \mathbf{r}_{c s}, t, \tau\right)\right] \\
& \times \widetilde{\Gamma}\left(\mathbf{r}_{c s}, \mathbf{q}^{\prime}, t, \tau\right) \mathrm{d}\left|\mathbf{r}_{c}-\mathbf{r}_{c s}\right| \mathrm{d} \Omega \mathrm{d} \mathbf{q}^{\prime} .
\end{aligned}
$$

Since the spectral density $\widetilde{\Gamma}\left(\mathbf{r}_{c s}, \mathbf{q}^{\prime}, t, \tau\right)$ of the quasiuniform mutual coherence function for quasimonochromatic light is approximately concentrated on a spherical shell with radius $\left|\mathbf{q}^{\prime}\right|=K_{r}[27,30,31,41,42]$, we relate the spectral density to the time-varying specific intensity $I\left(\mathbf{r}_{c s}, \hat{\mathbf{\Omega}}^{\prime}, t, \tau\right)$ by the following approximation $[27,28,30,31,41,42]$ :

$$
\widetilde{\Gamma}\left(\mathbf{r}_{c s}, \mathbf{q}^{\prime}, t, \tau\right) \approx \delta\left(\left|\mathbf{q}^{\prime}\right|-K_{r}\right) I\left(\mathbf{r}_{c s}, \hat{\mathbf{\Omega}}^{\prime}, t, \tau\right) / K_{r}^{2} .
$$

We can now combine Eqs. (8) and (15) to express the time-varying specific intensity as an angular spectrum of the mutual coherence function

$$
\begin{gathered}
\Gamma\left(\mathbf{r}_{c}, \mathbf{r}_{d}, t, \tau\right)=\int I\left(\mathbf{r}_{c}, \hat{\mathbf{\Omega}}, t, \tau\right) \exp \left(i K_{r} \hat{\mathbf{\Omega}} \cdot \mathbf{r}_{d}\right) \mathrm{d} \Omega, \\
\Gamma_{0}\left(\mathbf{r}_{c}, \mathbf{r}_{d}, t, \tau\right)=\int I_{0}\left(\mathbf{r}_{c}, \hat{\mathbf{\Omega}}, t, \tau\right) \exp \left(i K_{r} \hat{\mathbf{\Omega}} \cdot \mathbf{r}_{d}\right) \mathrm{d} \Omega,
\end{gathered}
$$

where Eq. (16b) is obtained by assuming that $\Gamma_{0}\left(\mathbf{r}_{c}, \mathbf{r}_{d}, t, \tau\right)$ satisfies expressions similar to Eqs. (8) and (15).
We can further use the time-varying specific intensity $I\left(\mathbf{r}_{c}, \hat{\mathbf{\Omega}}, t, \tau\right)$ to obtain the time-varying optical power spectral density $P\left(\mathbf{r}_{c}, t, \omega\right)$ of the ultrasound modulated light received in some solid angle $\Omega_{0}$ based on the Wigner distribution [43] as

$$
P\left(\mathbf{r}_{c}, t, \omega\right)=\frac{1}{2 \pi} \int_{-\infty}^{+\infty} I_{\Omega_{0}}\left(\mathbf{r}_{c}, t, \tau\right) \exp (i \omega \tau) \mathrm{d} \tau,
$$

where $I_{\Omega_{0}}\left(\mathbf{r}_{c}, t, \tau\right)=\int_{\Omega_{0}} I\left(\mathbf{r}_{c}, \hat{\mathbf{\Omega}}, t, \tau\right) \mathrm{d} \Omega$.

In the next step we substitute Eqs. (16a), (16b), and (15) into Eq. (14) and perform the integration over $\left|\mathbf{q}^{\prime}\right|$. After subsequently removing the integrals over $\hat{\boldsymbol{\Omega}}$ together with exponents $\exp \left(i K_{r} \hat{\mathbf{\Omega}} \cdot \mathbf{r}_{d}\right)$ that are common to all terms, we finally obtain the integral form of the CTE as

$$
\begin{aligned}
I(\mathbf{r}, \hat{\mathbf{\Omega}}, t, \tau)= & I_{0}(\mathbf{r}, \hat{\mathbf{\Omega}}, t, \tau)+\int \mu_{s} p\left(\hat{\mathbf{\Omega}}, \hat{\mathbf{\Omega}}^{\prime}\right) \\
& \times \exp \left(-\mu_{t}\left|\mathbf{r}-\mathbf{r}_{s}\right|\right) I\left(\mathbf{r}_{s}, \hat{\mathbf{\Omega}}^{\prime}, t, \tau\right) \\
& \times \Phi\left(\mathbf{r}, \mathbf{r}_{s}, \hat{\mathbf{\Omega}}, \hat{\mathbf{\Omega}}^{\prime}, t, \tau\right) \mathrm{d}\left|\mathbf{r}-\mathbf{r}_{s}\right| \mathrm{d} \Omega^{\prime}
\end{aligned}
$$

In Eq. (18), the redundant subscript $c$ was removed from the center-of-gravity coordinate $\mathbf{r}_{c}$, and we also assumed that $\mathbf{r}_{c s} \approx \mathbf{r}_{s}$. The factor

$$
\begin{aligned}
\Phi\left(\mathbf{r}, \mathbf{r}_{s}, \hat{\mathbf{\Omega}}, \hat{\mathbf{\Omega}}^{\prime}, t, \tau\right)= & \exp \left[i \Psi_{d}\left(\mathbf{r}_{s}, \hat{\mathbf{\Omega}}, \hat{\mathbf{\Omega}}^{\prime}, t, \tau\right)\right] \\
& \times \exp \left[i \Psi_{n}\left(\mathbf{r}, \mathbf{r}_{s}, t, \tau\right)\right]
\end{aligned}
$$

accounts for the ultrasound-induced optical phase increments due to both mechanisms of modulation. The term

$$
\Psi_{d}\left(\mathbf{r}_{s}, \hat{\mathbf{\Omega}}, \hat{\mathbf{\Omega}}^{\prime}, t, \tau\right)=-K_{r}\left(\hat{\mathbf{\Omega}}-\hat{\mathbf{\Omega}}^{\prime}\right) \cdot \Delta \mathbf{e}\left(\mathbf{r}_{s}, t, \tau\right)
$$

is due to ultrasound-induced displacement of the optical scatterers and is given by

$$
\begin{aligned}
\Psi_{d}\left(\mathbf{r}_{s}, \hat{\mathbf{\Omega}}, \hat{\mathbf{\Omega}}^{\prime}, t, \tau\right)= & \frac{K_{r} P_{0}}{\rho v_{a}}\left[\left(\hat{\mathbf{\Omega}}-\hat{\mathbf{\Omega}}^{\prime}\right) \cdot \hat{\mathbf{\Omega}}_{a}\right] \\
& \times \int_{t-\tau / 2}^{t+\pi / 2} h\left(t^{\prime}-v_{a} \hat{\mathbf{\Omega}}_{a} \cdot \mathbf{r}_{s}+\phi\right) \mathrm{d} t^{\prime},
\end{aligned}
$$

where we assume that optical scatterers follow the ultrasound-induced fluid movement in amplitude and phase [25].

We see that the time-varying specific intensity $I(\mathbf{r}, \hat{\mathbf{\Omega}}, t, \tau)$ in Eq. (18) is given as a sum of all time-varying specific intensities scattered into direction $\hat{\mathbf{\Omega}}$ including the unscattered term $I_{0}(\mathbf{r}, \hat{\mathbf{\Omega}}, t, \tau)$. Similar to the previously derived CTE for moving scatterers undergoing Brownian motion [35,38], in Eq. (18) we have a term $\Psi_{d}()$ that is due to the ultrasound-induced movement of the optical scatterers. In addition, in Eq. (18) we also have a new term $\Psi_{n}$ ( ) that is due to ultrasound-induced changes in the optical index of refraction.

Analytical solutions for $I(\mathbf{r}, \hat{\mathbf{\Omega}}, t, \tau)$ and $P(\mathbf{r}, t, \omega)$ are difficult to find from Eqs. (18) and (17) for any practical experimental configurations. Numerical codes and Monte 
Carlo algorithms, however, can be readily developed. In Section 3, one such Monte Carlo algorithm is presented.

\section{MONTE CARLO SIMULATION}

We developed a Monte Carlo (MC) algorithm that can be used to calculate the time-varying power spectral density of light modulated by a pulsed ultrasound field focused in an optically scattering medium with a heterogeneous distribution of optical parameters. We first divide the optically scattering medium into cells along the Cartesian axes and assign a vector $\mathbf{n}$ with integer coordinates $\left\{n_{x}, n_{y}, n_{z}\right\}$ to each cell. Next, we assign values of the optical absorption $\left(\mu_{a, \mathbf{n}}\right)$ and the scattering $\left(\mu_{s, \mathbf{n}}\right)$ coefficients as well as the scattering anisotropy factor $\left(g_{\mathbf{n}}\right)$ to each cell, where a Henyey-Greenstein scattering phase function is assumed [44]. The local parameters of the ultrasound field are also assigned to each cell: an average ultrasound propagation direction $\hat{\mathbf{\Omega}}_{a, \mathbf{n}}$, pressure amplitude $P_{0, \mathbf{n}}$, and time delay of the pulse $t_{\mathbf{n}}$. We assume that the dimensions of each cell are much smaller than the ultrasound wavelength and that the ultrasound field can be approximated within the cell as $P_{\mathbf{n}}(t)=P_{0, \mathbf{n}} h\left(t-t_{\mathbf{n}}\right)$, where $h(t)$ is the propagation function of the ultrasound pulse normalized to unity. We also assume that the error due to using the same propagation function $h(t)$ in all the cells is small. The procedure for propagation of the photon packets in the MC is the same as in [34], and similar to the previously described algorithms $[39,45]$. The photon packet is analyzed at each crossing of the cell boundaries, and the remaining length of the free path is adjusted based on the extinction coefficient within the cell that the photon packet is entering.

The trajectory of each photon consists of many small steps that are determined by all of the scattering events and cell boundaries along the way. For each small photon step of length $l_{k}$ within cell $\mathbf{m}_{k}$, we calculate the optical phase increment due to ultrasound-induced index of refraction changes as $\Delta \varphi_{n, k}(t)=k_{0} n_{0} l_{k} P_{\mathbf{m}_{b}}(t) \eta /\left(\rho v_{a}^{2}\right)$. Similarly, for each scattering event $j$ within cell $\mathbf{n}_{j}$, we calculate the optical phase increment that is due to ultrasound-induced scatterer displacement as

$$
\Delta \varphi_{d, j}(t)=k_{0} n_{0}\left[\left(\hat{\boldsymbol{\Omega}}_{\mathrm{inc}, j}-\hat{\mathbf{\Omega}}_{\mathrm{sc}, j}\right) \cdot \hat{\boldsymbol{\Omega}}_{a, \mathbf{n}_{j}}\right] A_{\mathbf{n}_{j}}(t),
$$

where $\hat{\mathbf{\Omega}}_{a, \mathbf{n}_{j}} A_{\mathbf{n}_{j}}(t)$ is the scatterer displacement vector and $\hat{\boldsymbol{\Omega}}_{\mathrm{inc}, j}$ and $\hat{\boldsymbol{\Omega}}_{\mathrm{sc}, j}$ are the incident and scattered photon directions, respectively.

At each scattering event, the total ultrasound-induced phase increment of the photon packet accumulated up to this point is $\Delta \varphi(t)=\Sigma_{k} \Delta \varphi_{n, k}(t)+\Sigma_{j} \Delta \varphi_{d, j}(t)$, and $k$ and $j$ count all of the previous steps and scattering events of the photon. We calculate the time-varying power spectral density $W_{p h} P(t, \omega)$ for the photon packet at time $t$ based on the Wigner distribution [43] as

$$
W_{p h} P(t, \omega)=W_{p h}(2 \pi)^{-1} \int_{-\infty}^{+\infty} W(t, \tau) \exp (i \omega \tau) \mathrm{d} \tau,
$$

where $W_{p h}$ is the current weight of a photon, $P(t, \omega)$ is the normalized time-varying power spectral density, and $W(t, \tau)$ is given by

$$
\begin{aligned}
W(t, \tau) & =\exp [i \Delta \varphi(t, \tau)], \\
\Delta \varphi(t, \tau) & =\Delta \varphi(t+\tau / 2)-\Delta \varphi(t-\tau / 2) .
\end{aligned}
$$

In Eq. (20), $\omega$ represents the angular frequency increment with respect to the angular frequency $\omega_{0}$ of the nonmodulated light.

We further assume that the difference in phase increment $\Delta \varphi(t, \tau)$ is small $(|\Delta \varphi(t, \tau)| \ll 1)$, and consider only the first three terms in the Taylor expansion of $W(t, \tau)$. The approximate expression for $P(t, \omega)$ from Eq. (20) is then given by

$$
P(t, \omega) \approx(2 \pi)^{-1} \int_{-\infty}^{+\infty}\left[1+i \Delta \varphi(t, \tau)-\frac{1}{2} \Delta \varphi^{2}(t, \tau)\right] \exp (i \omega \tau) \mathrm{d} \tau .
$$

We proceed by integrating the individual terms in Eq. (21). The phase increment difference $\Delta \varphi(t, \tau)$ is given by

$$
\begin{aligned}
\Delta \varphi(t, \tau)= & \frac{k_{0} n_{0} \eta}{\rho v_{a}^{2}} \sum_{k} l_{k} P_{0, \mathbf{m}_{k}}\left[h\left(t+\tau / 2-t_{\mathbf{m}_{k}}\right)-h\left(t-\tau / 2-t_{\mathbf{m}_{k}}\right)\right] \\
& +\frac{k_{0} n_{0}}{\rho v_{a}} \sum_{j}\left[\left(\hat{\boldsymbol{\Omega}}_{\mathrm{inc}_{j} j}-\hat{\mathbf{\Omega}}_{\mathrm{sc}_{j} j}\right) \cdot \hat{\boldsymbol{\Omega}}_{a, \mathbf{n}_{j}}\right] P_{0, \mathbf{n}_{j}} \\
& \times \int_{t-\tau / 2}^{t+\tau / 2} h\left(u-t_{\mathbf{n}_{j}}\right) \mathrm{d} u
\end{aligned}
$$

where we assumed that optical scatterers follow the ultrasound-induced fluid oscillations in amplitude and phase such that

$$
\left(\rho v_{a}\right)^{-1} P_{0, \mathbf{n}_{j}} \int_{t-\tau / 2}^{t+\tau / 2} h\left(u-t_{\mathbf{n}_{j}}\right) \mathrm{d} u=A_{\mathbf{n}_{j}}(t+\tau / 2)-A_{\mathbf{n}_{j}}(t-\tau / 2) .
$$

We denote with $P_{0}(t, \omega)$ the integral of the first term $\left[(2 \pi)^{-1} \int \exp (i \omega \tau) \mathrm{d} \tau \equiv \delta(\omega)\right]$ in Eq. (21). Thus, $W_{p h} P_{0}(t, \omega)$ contributes a value of $W_{p h}$ to the unmodulated intensity of light at time $t$.

The integral of the second term in Eq. (21) $\left[(2 \pi)^{-1} \int_{-\infty}^{+\infty} i \Delta \varphi(t, \tau) \exp (i \omega \tau) \mathrm{d} \tau\right]$ is then denoted with $P_{1}(t, \omega)$ and expressed as

$$
\begin{aligned}
P_{1}(t, \omega)= & -\frac{4 k_{0} n_{0} \eta}{\rho v_{a}^{2}} \sum_{k} l_{k} P_{0, \mathbf{m}_{k}} \operatorname{Im}\left[\widetilde{h}(2 \omega) \exp \left[-i 2 \omega\left(t-t_{\mathbf{m}_{k}}\right)\right]\right] \\
& -\frac{4 k_{0} n_{0}}{\rho v_{a}} \sum_{j} P_{0, \mathbf{n}_{j}}\left[\left(\hat{\mathbf{\Omega}}_{\mathrm{inc}_{j} j}-\hat{\mathbf{\Omega}}_{\mathrm{sc}_{j} j}\right) \cdot \hat{\mathbf{\Omega}}_{a, \mathbf{n}_{j}}\right] \\
& \times \operatorname{Re}\left[\frac{\tilde{h}(2 \omega)}{2 \omega} \exp \left[-i 2 \omega\left(t-t_{\mathbf{n}_{j}}\right)\right]\right.
\end{aligned}
$$

where $\widetilde{h}(\omega)$ is the Fourier transform of $h(t)$. It is physically meaningful to require that there be no dc component in the temporal spectrum of the ultrasonic pulse $[\widetilde{h}(0)$ $=0]$, which ensures that the expression for $P_{1}(t, \omega)$ in Eq. (23) be well defined for all angular frequencies $\omega$ and that $P_{1}(t, 0)=0$. Owing to the factor $\exp (2 i \omega t)$ in Eq. (23), $P_{1}(t, \omega)$ fluctuates fast in time. If $T_{a}$ is the time period associated with the central frequency of the ultrasound pulse, then the average value of $P_{1}(t, \omega)$ is approximately 
zero in any time interval that is comparable with or longer than $T_{a} / 2$, and we will neglect it in further derivations.

Finally, the more complex expression for the integral of the third term in Eq. (21) is given by

$$
P_{2}(t, \omega)=-\frac{1}{2} \frac{1}{2 \pi} \int_{-\infty}^{+\infty} \Delta \varphi^{2}(t, \tau) \exp (i \omega \tau) \mathrm{d} \tau
$$

Since

$$
\Delta \varphi(t, \tau)=\sum_{k} \Delta \varphi_{n, k}(t, \tau)+\sum_{j} \Delta \varphi_{d, j}(t, \tau)
$$

where

$$
\Delta \varphi_{n, k}(t, \tau)=\Delta \varphi_{n, k}(t+\tau / 2)-\Delta \varphi_{n, k}(t-\tau / 2)
$$

and

$$
\Delta \varphi_{d, j}(t, \tau)=\Delta \varphi_{d_{j} j}(t+\tau / 2)-\Delta \varphi_{d, j}(t-\tau / 2),
$$

we express $P_{2}(t, \omega)$ as

$$
P_{2}(t, \omega)=P_{2, n n}(t, \omega)+P_{2, d d}(t, \omega)+2 P_{2, n d}(t, \omega),
$$

where terms $P_{2, a b}(t, \omega)$ in Eq. (25) are given by

$$
P_{2, a b}(t, \omega)=-\frac{1}{2} \frac{1}{2 \pi} \int_{-\infty}^{+\infty} \sum_{k, j} \Delta \varphi_{a, k}(t, \tau) \Delta \varphi_{b, j}(t, \tau) \exp (i \omega \tau) \mathrm{d} \tau
$$

The integrals

$$
I_{a b, k, j}(t, \omega)=(2 \pi)^{-1} \int \Delta \varphi_{a, k}(t, \tau) \Delta \varphi_{b, j}(t, \tau) \exp (i \omega \tau) \mathrm{d} \tau
$$

of individual terms in Eq. (26) can be expressed as

$$
\begin{aligned}
I_{n n, k, j}( & t, \omega) \\
= & -4 \Lambda_{n}^{2} l_{k} l_{j} P_{0, \mathbf{m}_{k}} P_{0, \mathbf{n}_{j}}\left\{\operatorname { R e } \left[\exp \left[i \omega\left(t_{\mathbf{m}_{k}}-t_{\mathbf{n}_{j}}\right)\right]\right.\right. \\
& \left.\times \int_{-\infty}^{+\infty} \tilde{h}\left(\omega-\omega_{t}\right) \tilde{h}^{*}\left(\omega+\omega_{t}\right) \exp \left[i \omega_{t}\left(2 t-t_{\mathbf{m}_{k}}-t_{\mathbf{n}_{j}}\right)\right] \mathrm{d} \omega_{t}\right] \\
& -\operatorname{Re}\left[\exp \left[-i \omega\left(2 t-t_{\mathbf{m}_{k}}-t_{\mathbf{n}_{j}}\right)\right]\right. \\
& \left.\left.\times \int_{-\infty}^{+\infty} \tilde{h}\left(\omega-\omega_{t}\right) \tilde{h}\left(\omega+\omega_{t}\right) \exp \left[-i \omega_{t}\left(t_{\mathbf{m}_{k}}-t_{\mathbf{n}_{j}}\right)\right] \mathrm{d} \omega_{t}\right]\right\},
\end{aligned}
$$

$$
\begin{aligned}
& I_{d d, k, j}(t, \omega) \\
& =-4 \Lambda_{d}^{2} P_{0, \mathbf{m}_{k}} P_{0, \mathbf{n}_{j}}\left[\hat{\mathbf{\Omega}}_{a, \mathbf{m}_{k}} \cdot\left(\hat{\mathbf{\Omega}}_{\mathrm{inc}, k}-\hat{\boldsymbol{\Omega}}_{\mathrm{sc}, k}\right)\right] \\
& \quad \times\left[\hat{\boldsymbol{\Omega}}_{a, \mathbf{n}_{j}} \cdot\left(\hat{\boldsymbol{\Omega}}_{\mathrm{inc}_{j} j}-\hat{\mathbf{\Omega}}_{\mathrm{sc}, j}\right)\right]\left\{\operatorname { R e } \left[\exp \left[i \omega\left(t_{\mathbf{m}_{k}}-t_{\mathbf{n}_{j}}\right)\right]\right.\right. \\
& \left.\times \int_{-\infty}^{+\infty} \frac{\tilde{h}\left(\omega-\omega_{t}\right) \tilde{h}^{*}\left(\omega+\omega_{t}\right)}{\left(\omega-\omega_{t}\right)\left(\omega+\omega_{t}\right)} \exp \left[i \omega_{t}\left(2 t-t_{\mathbf{m}_{k}}-t_{\mathbf{n}_{j}}\right)\right] \mathrm{d} \omega_{t}\right] \\
& +\operatorname{Re}\left[\exp \left[-i \omega\left(2 t-t_{\mathbf{m}_{k}}-t_{\mathbf{n}_{j}}\right)\right]\right. \\
& \left.\left.\quad \times \int_{-\infty}^{+\infty} \frac{\tilde{h}\left(\omega-\omega_{t}\right) \tilde{h}\left(\omega+\omega_{t}\right)}{\left(\omega-\omega_{t}\right)\left(\omega+\omega_{t}\right)} \exp \left[-i \omega_{t}\left(t_{\mathbf{m}_{k}}-t_{\mathbf{n}_{j}}\right)\right] \mathrm{d} \omega_{t}\right]\right\},
\end{aligned}
$$

$$
I_{n d, k, j}(t, \omega)
$$

$$
=-4 \Lambda_{n} \Lambda_{d} l_{k} P_{0, \mathbf{m}_{k}} P_{0, \mathbf{n}_{j}}\left[\hat{\mathbf{\Omega}}_{a, \mathbf{n}_{j}} \cdot\left(\hat{\mathbf{\Omega}}_{\mathrm{inc}, j}-\hat{\boldsymbol{\Omega}}_{\mathrm{sc}, j}\right)\right]
$$

$$
\times\left\{\operatorname { I m } \left[\exp \left[i \omega\left(t_{\mathbf{m}_{k}}-t_{\mathbf{n}_{j}}\right)\right]\right.\right.
$$

$$
\begin{aligned}
& \left.\times \int_{-\infty}^{+\infty} \frac{\tilde{h}\left(\omega-\omega_{t}\right) \widetilde{h}^{*}\left(\omega+\omega_{t}\right)}{\left(\omega+\omega_{t}\right)} \exp \left[i \omega_{t}\left(2 t-t_{\mathbf{m}_{k}}-t_{\mathbf{n}_{j}}\right)\right] \mathrm{d} \omega_{t}\right] \\
& +\operatorname{Im}\left[\exp \left[-i \omega\left(2 t-t_{\mathbf{m}_{k}}-t_{\mathbf{n}_{j}}\right)\right]\right. \\
& \left.\left.\times \int_{-\infty}^{+\infty} \frac{\widetilde{h}\left(\omega-\omega_{t}\right) \tilde{h}\left(\omega+\omega_{t}\right)}{\omega+\omega_{t}} \exp \left[-i \omega_{t}\left(t_{\mathbf{m}_{k}}-t_{\mathbf{n}_{j}}\right)\right] \mathrm{d} \omega_{t}\right]\right\},
\end{aligned}
$$

where $\Lambda_{n}=k_{0} n_{0} \eta /\left(\rho v_{a}^{2}\right)$ and $\Lambda_{d}=k_{0} n_{0} /\left(\rho v_{a}\right)$.

The second terms in the curly brackets in Eqs. (27), (28), and (29) contain fast fluctuating factors $\exp [-i \omega(2 t$ $\left.-t_{\mathbf{m}_{k}}-t_{\mathbf{n}_{j}}\right)$, and we will neglect them in further derivations.

The difficulty of obtaining analytical solutions for the integrals in Eqs. (27), (28), and (29) depends on the particular form of the pulse propagation function $h(t)$. We choose a convenient but still general form of $h(t)$ as $h(t)$ $=\omega_{a}^{-1} \partial h_{0}(t) / \partial t$, where $h_{0}(t)=\exp \left[-t^{2} /\left(2 \sigma^{2} T_{a}^{2}\right)\right] \sin \left(\omega_{a} t\right)$ is a simple periodic function bounded with a Gaussian envelope. Here $T_{a}$ and $\omega_{a}=2 \pi / T_{a}$ are the temporal period and angular frequency of the sound wave associated with the central frequency of the pulse, respectively, and $\sigma$ is a nondimensional parameter that is used to control the temporal width of the pulse. The function $h(t)$ and its Fourier transform $\tilde{h}(\omega)$ are given by 


$$
h(t)=\exp \left(-\frac{t^{2}}{2\left(\sigma T_{a}\right)^{2}}\right)\left[\cos \left(\omega_{a} t\right)-\frac{t}{\omega_{a}\left(\sigma T_{a}\right)^{2}} \sin \left(\omega_{a} t\right)\right],
$$

$$
\begin{aligned}
\tilde{h}(\omega)= & \frac{1}{2} \frac{\omega}{\omega_{a} \sigma_{a} \sqrt{2 \pi}} \\
& \times\left[\exp \left(-\frac{\left(\omega+\omega_{a}\right)^{2}}{2 \sigma_{a}^{2}}\right)-\exp \left(-\frac{\left(\omega-\omega_{a}\right)^{2}}{2 \sigma_{a}^{2}}\right)\right],
\end{aligned}
$$

where $\sigma_{a}=1 /\left(\sigma T_{a}\right)$. The approximate values of the terms $I_{n n, k, j}(t, \omega), I_{d d, k, j}(t, \omega)$, and $I_{n d, k, j}(t, \omega)$, are then given by

$$
\begin{aligned}
I_{n n, k, j}(t, \omega)= & -\Lambda \eta^{2} v_{a}^{-2} l_{k} l_{j} P_{0, \mathbf{m}_{k}} P_{0, \mathbf{n}_{j}} \omega^{2} \Psi\left(t, t_{\mathbf{m}_{k}}, t_{\mathbf{n}_{j}}\right) \\
& \times \Xi(\omega) \cos \left[\omega\left(t_{\mathbf{m}_{k}}-t_{\mathbf{n}_{j}}\right)\right], \\
I_{d d, k, j}(t, \omega)= & -\Lambda P_{0, \mathbf{m}_{k}} P_{0, \mathbf{n}_{j}}\left[\hat{\mathbf{\Omega}}_{a, \mathbf{m}_{k}} \cdot\left(\hat{\boldsymbol{\Omega}}_{\mathrm{inc}, k}-\hat{\mathbf{\Omega}}_{\mathrm{sc}, k}\right)\right] \\
& \times\left[\hat{\mathbf{\Omega}}_{a, \mathbf{n}_{j}} \cdot\left(\hat{\boldsymbol{\Omega}}_{\mathrm{inc}, j}-\hat{\mathbf{\Omega}}_{\mathrm{sc}, j}\right)\right] \\
& \times \Psi\left(t, t_{\mathbf{m}_{k}}, t_{\mathbf{n}_{j}}\right) \Xi(\omega) \cos \left[\omega\left(t_{\mathbf{m}_{k}}-t_{\mathbf{n}_{j}}\right)\right], \quad(33) \\
I_{n d, k, j}(t, \omega)= & -\Lambda \eta v_{a}^{-1} l_{k} P_{0, \mathbf{m}_{k}} P_{0, \mathbf{n}_{j}}\left[\hat{\mathbf{\Omega}}_{a, \mathbf{n}_{j}} \cdot\left(\hat{\boldsymbol{\Omega}}_{\mathrm{inc}_{j} j}-\hat{\mathbf{\Omega}}_{\mathrm{sc}, j}\right)\right] \\
& \times \Psi\left(t, t_{\mathbf{m}_{k}}, t_{\mathbf{n}_{j}}\right) \Xi(\omega)\left(\omega \sin \left[\omega\left(t_{\mathbf{m}_{k}}-t_{\mathbf{n}_{j}}\right)\right]\right. \\
& \left.-\sigma_{a}^{2}\left(t-\frac{t_{\mathbf{m}_{k}}+t_{\mathbf{n}_{j}}}{2}\right) \cos \left[\omega\left(t_{\mathbf{m}_{k}}-t_{\mathbf{n}_{j}}\right)\right]\right),
\end{aligned}
$$

where

$$
\begin{gathered}
\Lambda=\left(k_{0} n_{0}\right)^{2} /\left[2\left(\rho v_{a}\right)^{2} \omega_{a}^{2} \sigma_{a} \sqrt{\pi}\right], \\
\Psi\left(t, t_{\mathbf{m}_{k}}, t_{\mathbf{n}_{j}}\right)=\exp \left[-\sigma_{a}^{2}\left(t-t_{\mathbf{m}_{k}} / 2-t_{\mathbf{n}_{j}} / 2\right)^{2}\right], \\
\Xi(\omega)=\exp \left[-\left(\omega+\omega_{a}\right)^{2} / \sigma_{a}^{2}\right]+\exp \left[-\left(\omega-\omega_{a}\right)^{2} / \sigma_{a}^{2}\right] .
\end{gathered}
$$

Let $M$ and $N_{M}$ represent the total number of scattering events and the total number of free steps, respectively, of the photon at scattering event $M$ that happens at time $t$. Since we analyze the photon propagation at each cell boundary, the number of small free steps $N_{M}$ can be greater than $M$. We calculate the value of $P_{2}(t, \omega)$ associated with the photon at each scattering event $M$ as

$$
\begin{aligned}
P_{2}(t, \omega)= & -\frac{1}{2} \sum_{k=1}^{N_{M}} \sum_{j=1}^{N_{M}} I_{n n, k, j}(t, \omega)-\frac{1}{2} \sum_{k=1}^{M} \sum_{j=1}^{M} I_{d d, k, j}(t, \omega) \\
& -\sum_{k=1}^{N_{M}} \sum_{j=1}^{M} I_{n d, k, j}(t, \omega) .
\end{aligned}
$$

We specify a discrete set of times $t_{s}$ during the time of flight of the ultrasonic pulse in the scattering sample, and a discrete set of angular frequencies $\omega_{q}$ around the central angular frequency of the ultrasound pulse $\omega_{a}$. At every scattering event of the photon, quantity $P_{2}\left(t_{s}, \omega_{q}\right)$ is calculated for all $t_{s}$ and $\omega_{q}$, and $\Delta P_{2, \mathbf{m}}\left(t_{s}, \omega_{q}\right)$ $=P_{2}\left(t_{s}, \omega_{q}\right) \Delta W_{p h}$ is evaluated, where $\Delta W_{p h}=W_{p h} \mu_{a, \mathbf{m}} / \mu_{t, \mathbf{m}}$, and $W_{p h}$ is the current weight of a photon at the scattering event that occurs in cell $\mathbf{m}$. At the end of the simulation of all of the photon packets, sums $P_{2, \mathbf{m}}\left(t_{s}, \omega_{q}\right)$ $=\Sigma \Delta P_{2, \mathbf{m}}\left(t_{s}, \omega_{q}\right)$ of the increments for all of the scattering events that have happened in cell $\mathbf{m}$ are proportional to the time-varying power spectral density of light at time $t_{s}$ and angular frequency $\omega_{q}$.

The sample in our simulation is an optically scattering slab with a thickness of $20 \mathrm{~mm}$ along the $X$ axis (Fig. 1). The dimensions of the slab in the $Y$ and $Z$ directions are both $100 \mathrm{~mm}$, which minimizes the error of the simulation within the central region. We define the background optical properties of the scattering slab as $\mu_{a}=0.1 \mathrm{~cm}^{-1}$ and $\mu_{s}=10 \mathrm{~cm}^{-1}$ in the entire slab, which values are representative of soft biological tissue for visible and nearinfrared light, and, for simplicity, assume isotropic scattering. An ultrasound pulse propagates parallell to the $Z$ axis within the slab, spaced at equal distances from the slab surfaces. The temporal profile of the pulse is given by Eq. (30), with the central frequency of $1 \mathrm{MHz}$ and $\sigma$ $=0.65$. The focal spot of the transducer coincides with the center of the slab $(\{x, y, z\}=\{10 \mathrm{~mm}, 0 \mathrm{~mm}, 0 \mathrm{~mm}\})$, and the peak pressure amplitude $P_{0}$ at the focus is $10^{5} \mathrm{~Pa}$. In order to model the ultrasound field we define the concave transducer element with an aperture diameter of $25.4 \mathrm{~mm}$ and a focal length of $40 \mathrm{~mm}$. The distributions of the ultrasound pressure and phase are calculated with the publicly available software FIELD II [46] versus time $t_{s}$ equally spaced by $0.5 \mu \mathrm{s}$. The ultrasound propagation directions are subsequently obtained by taking the gradient of the ultrasound phase. A pencil light source with a wavelength of $532 \mathrm{~nm}$ irradiates the scattering slab from the $\quad x<0$ half space at position $\{x, y, z\}$ $=\{0 \mathrm{~mm}, 0 \mathrm{~mm}, 0 \mathrm{~mm}\}$. We assume the same optical index of refraction $n_{0}=1.33$ in whole space, a mass density of the medium $\rho=10^{3} \mathrm{~kg} \mathrm{~m}^{-3}$, an ultrasound velocity $v_{a}$ $=1480 \mathrm{~m} \mathrm{~s}^{-1}$, and an elasto-optical coefficient of water at room temperature $\eta=0.32$. The dimensions of the cells that divide the simulation volume are $\Delta x=0.5 \mathrm{~mm}, \Delta y$ $=0.5 \mathrm{~mm}$, and $\Delta z=0.1 \mathrm{~mm}$, such that the change in ultra-

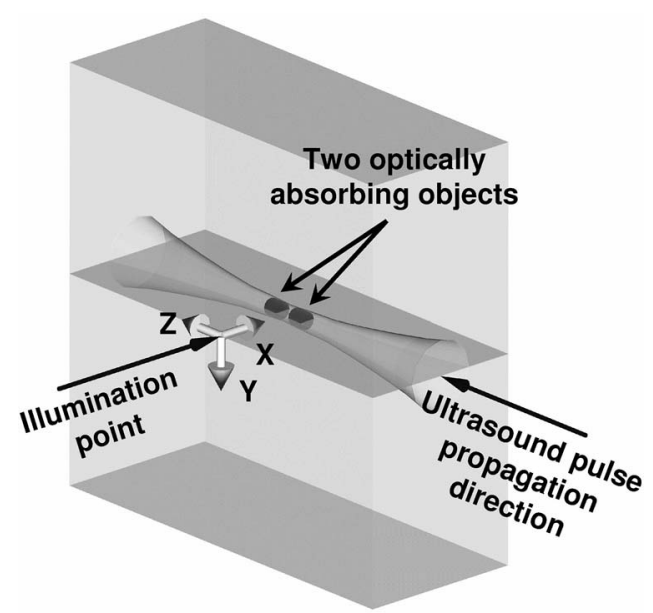

Fig. 1. Configuration of the scattering sample. Dimensions in the $X, Y$, and $Z$ directions are $20 \mathrm{~mm}, 100 \mathrm{~mm}$, and $100 \mathrm{~mm}$, respectively. 

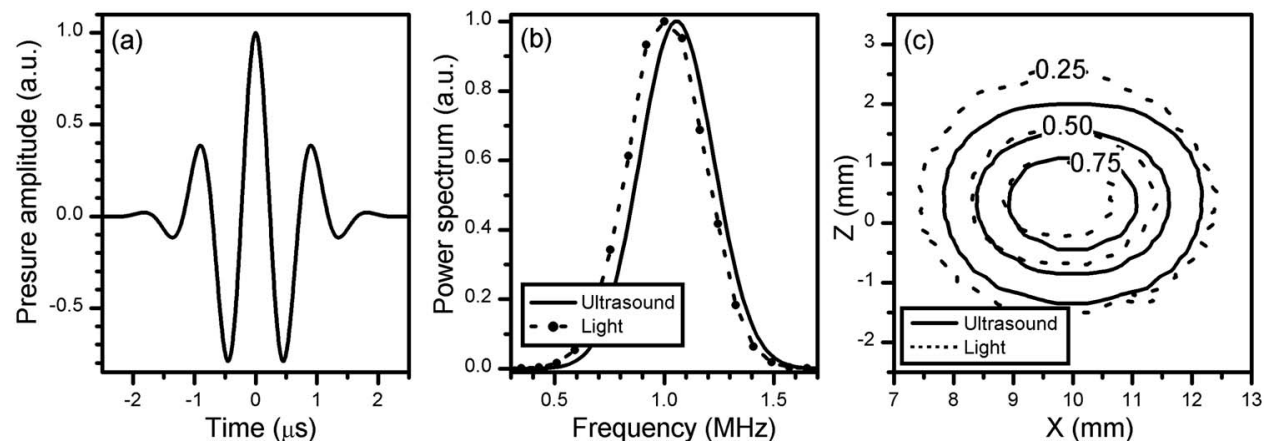

Fig. 2. (a) Temporal profile of the ultrasound pulse. (b) Power spectrum of the ultrasound pressure (solid curve) calculated from (a) and time-varying power spectrum of the ultrasound-modulated light (dotted-dashed curve) simulated at the transmission side of the slab at $\{x, y, z\}=\{20 \mathrm{~mm}, 0 \mathrm{~mm}, 0 \mathrm{~mm}\}$. The simulation results are shown for the ultrasound pulse at the focal point. (c) Contour plot of the spatial distributions of the ultrasonic power density (solid curve) and the time-varying power density (dashed curve) of the ultrasoundmodulated light. The distributions are presented in the plane defined by $y=0 \mathrm{~mm}$, for ultrasound pulse at the focal point. The contours are plotted at $25 \%, 50 \%$, and $75 \%$ of the maximum power levels. In this simulation no absorbing objects are present in the scattering slab.

sound phase within each cell is small. The $\Delta P_{2, \mathbf{m}}\left(t_{s}, \omega_{q}\right)$ values are sampled at discrete frequencies $\omega_{q}=2 \pi f_{q}$, where $f_{q}$ is equally spaced by $\Delta f_{q} \approx 82 \mathrm{kHz}$ from $265 \mathrm{kHz}$ to $1.74 \mathrm{MHz}$. In order to reduce the memory requirement, the values of $\Delta P_{2, \mathbf{m}}\left(t_{s}, \omega_{q}\right)$ are recorded only in cells $\mathbf{m}$ that belong to the plane defined by $y=0 \mathrm{~mm}$.

In Fig. 2(a), we present the temporal profile of the ultrasound pressure given by $h(t)$. Figure 2(b) shows the power spectrum of the ultrasound pressure (solid curve) calculated from $h(t)$, and the time-varying power spectrum (dotted-dashed curve) of the ultrasound-modulated light simulated at the transmission side of the slab at $\{x, y, z\}=\{20 \mathrm{~mm}, 0 \mathrm{~mm}, 0 \mathrm{~mm}\}$. In this simulation, the absorbing objects from Fig. 1 are not present in the scattering slab and the results of simulation are shown for the ultrasound pulse at the focal point. It was previously suggested by the theoretical model based on the DWS approach [25] that the efficiency of modulation of light by ultrasound in optically turbid media is smaller at the higher ultrasound frequencies. Therefore, in Fig. 2(b) the time-varying power spectral density of the ultrasoundmodulated light has a lower center frequency than that of the ultrasound pulse.

Figure 2(c) presents the contour plots of the spatial distributions of the ultrasonic power density (solid curve) and the time-varying power density (dashed curve) of the ultrasound-modulated light. The distributions are presented in the plane defined by $y=0 \mathrm{~mm}$, for an ultrasound pulse at the focal point. The contours are plotted at $25 \%$, $50 \%$, and $75 \%$ of the maximum power density levels. The values of the time-varying power densities of the ultrasound-modulated light in each cell $\mathbf{m}$ are calculated from $P_{2, \mathbf{m}}\left(t_{s}, \omega_{q}\right)$ by multiplying with $2 \pi \Delta f_{q} \mu_{a, \mathbf{m}}$ the sum of all of the $\omega_{q}$ spectral components around the central frequency $\omega_{a}$ and by subsequently multiplying the obtained value by 2 to account for both first-order sidebands of the modulated light. Similarly, the values of the ultrasonic power densities are obtained by integrating the power spectral density of the ultrasound pressure. There is a very good match between the $50 \%$ power density levels in the two distributions, which indicates that the spatial distribution of the 'virtual source' of ultrasoundmodulated light within the optically scattering sample is strongly correlated with the spatial distribution of the ultrasonic power density. In addition, distribution of the time-varying power density of the ultrasound-modulated light is extended toward the point of optical illumination of the sample. This anticipated behavior indicates the influence of distribution of the optical fluence in the sample on the spatial distribution of the 'virtual source' of ultrasound-modulated light.

Next, we position two optically absorbing cylinders $\left(\mu_{a}=100 \mathrm{~cm}^{-1}\right)$, both $2 \mathrm{~mm}$ in diameter and $2 \mathrm{~mm}$ long, within the scattering sample. The axes of both cylinders are parallel to the propagation direction of the ultrasound pulse. The separation between the absorbing objects is $2 \mathrm{~mm}$ and the center of separation coincides with the focal point of the ultrasound pulse $(\{x, y, z\}$ $=\{10 \mathrm{~mm}, 0 \mathrm{~mm}, 0 \mathrm{~mm}\})$. In Fig. 3 , we show one frame of the time-varying power density of the ultrasoundmodulated light simulated for the duration of propagation of the ultrasound pulse in the scattering sample. The consecutive frames in the multimedia online version of Fig. 3 are equally spaced by $0.5 \mu$ s time intervals, and in each frame the spatial distribution of the power density is presented in the plane defined by $y=0 \mathrm{~mm}$. The values are presented in shades of gray, with the levels equally spaced from zero to the maximum value. The maximum value of distribution $\left(I_{m}\right)$ in each frame is given in the upper right corner. The intensity of the ultrasoundmodulated light is much higher when the ultrasound pulse is close to the focal point. The distribution of ultrasound-modulated light has sharp boundaries at the edges of absorbing objects. In addition, overlap of the ultrasound pulse with the position of either one of the absorbing objects causes a very significant decay in the intensity of ultrasound-modulated light that is generated.

In Fig. 4, we present the temporal profile of modulation depth $M(t)$ of ultrasound-modulated light when the ultrasound pulse propagates through the scattering slab with two absorbing objects. The modulation depth is calculated as a ratio of the time-varying power density of ultrasound-modulated light to the power density of unmodulated light (proportional to the sum of $\Delta W_{p h} \mu_{a, \mathbf{m}}$ values for all of the scattering events in cell $\mathbf{m})$. Since $M(t)$ $\ll 1$, we assume that the error due to considering the op- 
tical fluence rate of unmodulated light equal to the total optical fluence rate is small for the purpose of calculating $M(t)$. The solid curve in Fig. 4 represents the case when the detection point is placed at $\{x, y, z\}$ $=\{20 \mathrm{~mm}, 0 \mathrm{~mm}, 0 \mathrm{~mm}\}$-at the transmission plane of the slab and symmetrically with respect to both the illumination beam and the two objects. This is a common experimental configuration that usually provides good signal-to-

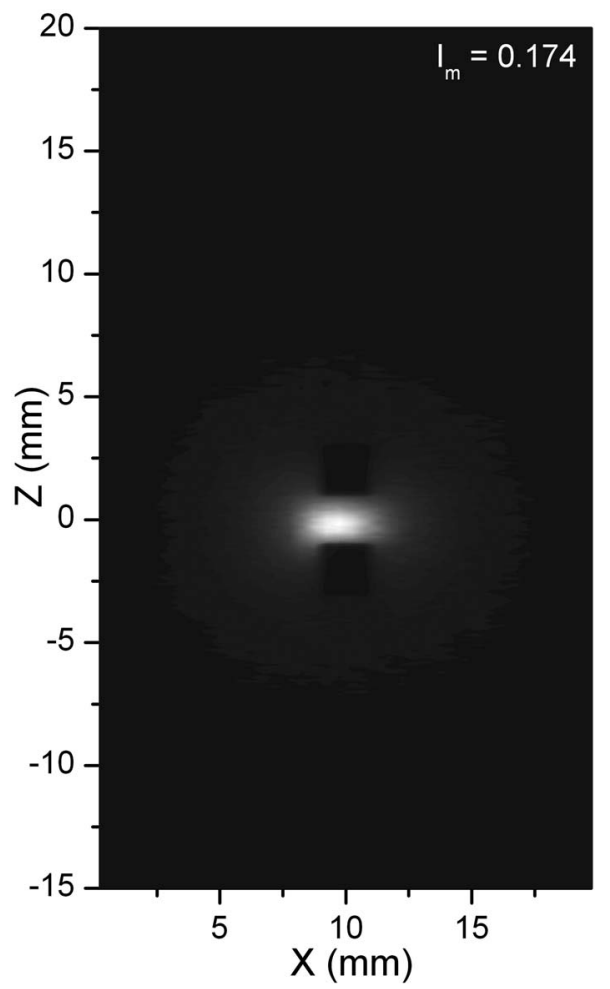

Fig. 3. (Multimedia online; josaa.osa.org) Static frame of the time-varying power density of the ultrasound-modulated light simulated for the duration of time-of-flight of the ultrasound pulse in the scattering sample. The spatial distribution of the power density is presented in the plane defined by $y=0 \mathrm{~mm}$. The values are presented in shades of gray, with the levels equally spaced from zero to the maximum value. The maximum value of distribution $\left(I_{m}\right)$ in each frame is given in the upper right corner.

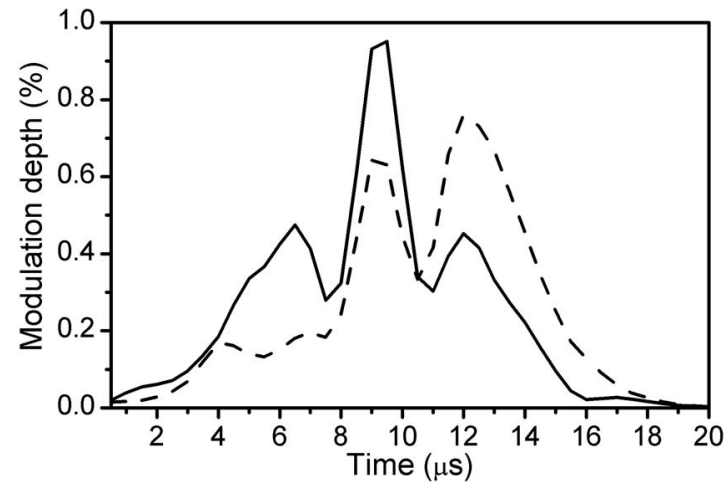

Fig. 4. Modulation depth simulated at the transmission plane of the scattering sample during the ultrasound pulse propagation. Solid curve, the case when the detection point is placed at $\{x, y, z\}=\{20 \mathrm{~mm}, 0 \mathrm{~mm}, 0 \mathrm{~mm}\}$-symmetrically with respect to both the illumination beam and the two objects. Dashed curve, the case when the detection point is placed at $\{x, y, z\}$ $=\{20 \mathrm{~mm}, 0 \mathrm{~mm}, 3 \mathrm{~mm}\}-3 \mathrm{~mm}$ away from the previous detection point. noise ratio, resolution, and contrast. The maximum modulation depth of $\approx 1 \%$ is obtained when the ultrasound pulse is at the focal point. There are two distinctive minima in the modulation depth profile that correlate with the times when the ultrasound pulse overlaps with the absorbing objects, which indicates the potential of UOT for high-contrast and high-resolution imaging. However, breaking the symmetry between the positions of illumination, detection, and the objects (dashed curve in Fig. 4) leads to a significant change in the profile of the modulation depth that is detected. This underlines the influence of the configuration of the imaging sample together with positions of the sources and detectors on the detected signal. It seems reasonable to expect that multiple positions of sources and detectors would be necessary to obtain an image of a complex sample.

\section{CONCLUSION}

In conclusion, based on the ladder approximation of the Bethe-Salpeter equation and Wigner time-frequency distribution, we have developed an integral form of the CTE for multiply scattered light modulated by an ultrasound pulse. The derivations are valid under the weakscattering approximation within the medical ultrasound frequency range and under moderate ultrasound pressures, and further theoretical development should address the setups with highly focused ultrasound and with very high ultrasound pressure. We have also developed a Monte Carlo algorithm that can be used to calculate the time-varying power spectral density of light modulated by the focused ultrasound pulse in optically turbid media with heterogeneous distributions of optical parameters. This permitted us, for what we think to be the first time, to obtain the spatial distribution of the ultrasoundmodulated light intensity during ultrasound pulse propagation in a scattering sample that contains optically absorbing objects. We expect the CTE and the MC algorithm to be of use for the estimation of resolution, contrast, and signal-to-noise ratios in UOT.

\section{ACKNOWLEDGMENT}

This project was sponsored by National Institutes of Health grant R33 CA094267.

\section{REFERENCES}

1. A. P. Gibson, J. C. Hebden, and S. R. Arridge, "Recent advances in diffuse optical imaging," Phys. Med. Biol. 50, R1-R43 (2005)

2. F. A. Marks, H. W. Tomlinson, and G. W. Brooksby, "A comprehensive approach to breast cancer detection using light: photon localization by ultrasound modulation and tissue characterization by spectral discrimination," in Proc. SPIE 1888, 500-510 (1993).

3. L. V. Wang, S. L. Jacques, and X. Zhao, "Continuous-wave ultrasonic modulation of scattered laser light to image objects in turbid media," Opt. Lett. 20, 629-631 (1995).

4. S. Sakadzic and L. V. Wang, "High-resolution ultrasoundmodulated optical tomography in biological tissues," Opt. Lett. 29, 2770-2772 (2004).

5. C. Kim, R. J. Zemp, and L. V. Wang, "Intense acoustic bursts as a signal-enhancement mechanism in ultrasoundmodulated optical tomography," Opt. Lett. 31, 2423-2425 (2006). 
6. M. Kempe, M. Larionov, D. Zaslavsky, and A. Z. Genack, "Acousto-optic tomography with multiply scattered light," J. Opt. Soc. Am. A 14, 1151-1158 (1997).

7. L. V. Wang and G. Ku, "Frequency-swept ultrasoundmodulated optical tomography of scattering media," Opt. Lett. 23, 975-977 (1998).

8. S. Leveque, A. C. Boccara, M. Lebec, and H. Saint-Jalmes, "Ultrasonic tagging of photon paths in scattering media: parallel speckle modulation processing," Opt. Lett. 24, 181-183 (1999).

9. G. Yao, S.-L. Jiao, and L. V. Wang, "Frequency-swept ultrasound-modulated optical tomography in biological tissue by use of parallel detection," Opt. Lett. 25, 734-736 (2000)

10. A. Lev, Z. Kotler, and B. G. Sfez, "Ultrasound tagged light imaging in turbid media in a reflectance geometry," Opt. Lett. 25, 378-380 (2000).

11. M. Hisaka, T. Sugiura, and S. Kawata, "Optical crosssectional imaging with pulse ultrasound wave assistance," J. Opt. Soc. Am. A 18, 1531-1534 (2001).

12. J. Li, G. $\mathrm{Ku}$, and L. V. Wang, "Ultrasound-modulated optical tomography of biological tissue by use of contrast of laser speckles,” Appl. Opt. 41, 6030-6035 (2002).

13. A. Lev and B. G. Sfez, "Pulsed ultrasound-modulated light tomography," Opt. Lett. 28, 1549-1551 (2003).

14. M. Gross, P. Goy, and M. Al-Koussa, "Shot-noise detection of ultrasound-tagged photons in ultrasound-modulated optical imaging," Opt. Lett. 28, 2482-2484 (2003).

15. T. W. Murray, L. Sui, G. Maguluri, R. A. Roy, A. Nieva, F. Blonigen, and C. A. DiMarzio, "Detection of ultrasound modulated photons in diffuse media using the photorefractive effect," Opt. Lett. 29, 2509-2511 (2004).

16. A. Lev, E. Rubanov, B. Sfez, S. Shany, and A. J. Foldes, "Ultrasound-modulated light tomography assessment of osteoporosis," Opt. Lett. 30, 1692-1694 (2005).

17. E. Bossy, L. Sui, T. W. Murray, and R. A. Roy, "Fusion of conventional ultrasound imaging and acousto-optic sensing by use of a standard pulsed-ultrasound scanner," Opt. Lett. 30, 744-746 (2005).

18. W. Leutz and G. Maret, "Ultrasonic modulation of multiply scattered-light," Physica B 204, 14-19 (1995).

19. G. Maret and P. E. Wolf, "Multiple light-scattering from disordered media-the effect of Brownian-motion of scatterers," Z. Phys. B: Condens. Matter 65, 409-413 (1987)

20. G. D. Mahan, W. E. Engler, J. J. Tiemann, and E. G. Uzgiris, "Ultrasonic tagging of light: Theory," in Proc. Natl. Acad. Sci. U.S.A. 95, 14015-14019 (1998).

21. L. V. Wang, "Mechanisms of ultrasonic modulation of multiply scattered coherent light: an analytic model," Phys. Rev. Lett. 87, 043903-(1-4) (2001).

22. D. J. Pine, D. A. Weitz, P. M. Chaikin, and E. Herbolzheimer, "Diffusing-wave spectroscopy," Phys. Rev. Lett. 60, 1134-1137 (1988).

23. S. Sakadzic and L. V. Wang, "Ultrasonic modulation of multiply scattered coherent light: an analytical model for anisotropically scattering media," Phys. Rev. E 66, 026603-(1-19) (2002).

24. A. Lev and B. Sfez, "In vivo demonstration of the ultrasound modulated light technique," J. Opt. Soc. Am. A 20, 2347-2354 (2003)

25. S. Sakadzic and L. V. Wang, "Modulation of multiply scattered coherent light by ultrasonic pulses: An analytical model," Phys. Rev. E 72, 036620-(1-12) (2005).

26. U. Frisch, Wave Propagation in Random Media, Vol. 1, Probabilistic Methods in Applied Mathematics (Academic, 1968), pp. 75-198.
27. Y. N. Barabanenkov, A. G. Vinogradov, Yu. A. Kravtsov, and V. I. Tatarskii, "Application of the theory of multiple scattering of waves to the derivation of the radiation transfer equation for a statistically inhomogeneous medium," Radiophys. Quantum Electron. 15, 1420-1425 (1972).

28. A. Ishimaru, Wave Propagation and Scattering in Random Media (Academic, 1978).

29. M. C. W. van Rossum, and Th. M. Nieuwenhuizen, "Multiple scattering of classical waves: microscopy, mesoscopy, and diffusion," Rev. Mod. Phys. 71, 313-371 (1999).

30. Yu. A. Kravtsov and L. A. Apresyan, "Radiative transfer: new aspects of the old theory," Vol. 36, Progress in Optics, E. Wolf, ed. (Elsevier, 1996), pp. 179-244.

31. S. M. Rytov, Yu. A. Kravtsov, and V. I. Tatarskii, Principles of Statistical Radiophysics 4: Wave Propagation through Random Media, 1st ed. (Springer Verlag, 1989).

32. V. L. Kuzmin and V. P. Romanov, "Coherent phenomena in light scattering from disordered systems," Phys. Usp. 39, 231-260 (1996).

33. S. Sakadzic and L. V. Wang, "Correlation transfer and diffusion of ultrasound-modulated multiply scattered light," Phys. Rev. Lett. 96, 163902-(1-4) (2006).

34. S. Sakadzic and L. V. Wang, "Correlation transfer equation for ultrasound-modulated multiply scattered light," Phys. Rev. E 74, 036618-(1-10) (2006).

35. A. Ishimaru, "Correlation functions of a wave in a random distribution of stationary and moving scatterers," Radio Sci. 10, 45-52 (1975).

36. M. J. Stephen, "Temporal fluctuations in wave propagation in random media," Phys. Rev. B 37, 1-5 (1988).

37. F. C. MacKintosh and J. Sajeev, "Diffusing-wave spectroscopy and multiple scattering of light in correlated random media," Phys. Rev. B 40, 2383-2406 (1989).

38. R. L. Dougherty, B. J. Ackerson, N. M. Reguigui, F. DorriNowkoorani, and U. Nobbmann, "Correlation transfer: development and application,” J. Quant. Spectrosc. Radiat. Transf. 52, 713-727 (1994).

39. L. V. Wang, "Mechanisms of ultrasonic modulation of multiply scattered coherent light: a Monte Carlo model," Opt. Lett. 26, 1191-1193 (2001).

40. G. Yao and L. Wang, "Signal dependence and noise source in ultrasound-modulated optical tomography," Am. J. Optom. Physiol. Opt. 43, 1320-1326 (2004).

41. Y. N. Barabanenkov, "Application of the smoothperturbation method to the solution of general equations of multiple wave-scattering theory," Sov. Phys. JETP 27, 954-959 (1968).

42. S. M. Rytov, Yu. A. Kravtsov, and V. I. Tatarskii, Principles of Statistical Radiophysics 3: Elements of Random Fields, 1st ed. (Springer Verlag, 1989).

43. L. Cohen, "Time-frequency distributions-A review," Proc. IEEE 77, 941-981 (1989).

44. L. G. Henyey and J. L. Greenstein, "Diffuse radiation in the Galaxy," Astrophys. J. 93, 70-83 (1941).

45. L. V. Wang, S. L. Jacques, and L.-Q. Zheng, "MCML-Monte Carlo modeling of photon transport in multi-layered tissues," Comput. Methods Programs Biomed. 47, 131-146 (1995)

46. J. A. Jensen and N. B. Svendsen, "Calculation of pressure fields from arbitrarily shaped, apodized, and excited ultrasound transducers," IEEE Trans. Ultrason. Ferroelectr. Freq. Control 39, 262-267 (1992). 